\title{
Energy Efficiency Optimization for NOMA with SWIPT
}

\author{
Jie Tang, Senior Member, IEEE, Jingci Luo, Mingqian Liu, Member, IEEE, Daniel So, Senior Member, IEEE, \\ Emad Alsusa, Senior Member, IEEE, Gaojie Chen, Senior Member, IEEE, Kai-Kit Wong, Fellow, IEEE, \\ and Jonathon Chambers, Fellow, IEEE
}

\begin{abstract}
The combination of simultaneous wireless information and power transfer (SWIPT) and non-orthogonal multiple access (NOMA) is a potential solution to improve spectral efficiency (SE) and energy efficiency (EE) of the upcoming fifth generation (5G) networks, especially in order to support the functionality of the Internet of things (IoT) and the massive machine-type communications (mMTC) scenarios. In this paper, we investigate joint power allocation and time switching (TS) control for EE optimization in a TS-based SWIPT NOMA system. Our aim is to optimize the EE of the system whilst satisfying the constraints on maximum transmit power budget, minimum data rate and minimum harvested energy per-terminal. The considered EE optimization problem is neither linear nor convex involving joint optimization of power allocation and time switching factors, and thus is extremely difficult to solve directly. In order to tackle this problem, we develop a dual-layer algorithm where Dinkelbach method is employed both in the inner-layer to optimize the power allocation and in the outer-layer to control the time switching assignment. Furthermore, a simplified but practical special case with equal time switching factors in all terminals is considered. Numerical results validate the theoretical findings and demonstrate that significant performance gain over orthogonal multiple access (OMA) scheme in terms of EE can be achieved by the proposed algorithms in a SWIPT-enabled NOMA system.
\end{abstract}

Index Terms-Energy efficiency (EE), non-orthogonal multiple access (NOMA), simultaneous wireless information and power transfer (SWIPT), time switching (TS).

\section{INTRODUCTION}

The exponential growth of wireless data services driven by mobile internet and connected devices has triggered the

This paper was presented in part at the IEEE Global Communications Conference (GLOBECOM), Abu Dhabi, UAE, 2018. This work has been supported in part by the National Natural Science Foundation of China under Grant 61601186, in part by the Natural Science Foundation of Guangdong Province under Grant 2017A030313383, in part by the Guangzhou Science Technology and Innovation Commission under Grant 201707010159, and in part by the Open Research Fund of National Mobile Communications Research Laboratory, Southeast University (No. 2019D06). (Corresponding author: Mingqian Liu.)

J. Tang and J. Luo are with the School of Electronic and Information Engineering, South China University of Technology, Guangzhou, China. (email: eejtang@scut.edu.cn; 201720110615@mail.scut.edu.cn).

M. Liu is with the State Key Laboratory of Integrated Services Networks, Xidian University, China. (e-mail: mqliu@ mail.xidian.edu.cn)

D. K. C. So and E. Alsusa are with the School of Electrical and Electronic Engineering, University of Manchester, Manchester, United Kingdom. (e-mail: d.so@manchester.ac.uk; e.alsusa@manchester.ac.uk).

K.-K Wong is with the Department of Electronic and Electrical Engineering, University College London, London, United Kingdom. (e-mail: kaikit.wong@ucl.ac.uk).

G. Chen and J. Chambers are with the Department of Engineering, University of Leicester, United Kingdom. (email: gaojie.chen@leicester.ac.uk; jonathon.chambers@leicester.ac.uk). investigation of fifth generation (5G) cellular networks. However, the available spectrum resources are far from enough to support the communication systems with the increasing demand for high data rate. This trend makes spectral efficiency (SE) to be the main performance indicator for the design and optimization of wireless systems, but at the same time constitutes to ever-rising network power consumption which has severe implications in terms of both economic and ecological costs, and thus energy saving has been recognized as an urgent issue worldwide. In order to meet these requirements, the evolving $5 \mathrm{G}$ cellular wireless networks are envisioned to provide higher efficiency of resource utilization, including both SE and energy efficiency (EE) [1].

Orthogonal frequency division multiple access (OFDMA) has served as the multiple access scheme in $4 \mathrm{G}$ owing to its significant performance against multipath fading as well as its higher SE compared to the previously used multiple access schemes [2]. However, in an OFDMA-based system, each subcarrier is only occupied by a single terminal in spite of the channel conditions, which limits the achievable SE. On the other hand, non-orthogonal multiple access (NOMA) can further improve the SE as each subcarrier is allowed to serve multiple terminals at the same time [3], and hence it has received considerable attention as a promising candidate for 5G [4]-[9]. Sharing the same spectrum among users leads to a high mutual interference when decoding information. However, by employing successive interference cancellation (SIC) at the receivers, the information can be correctly decoded and thus improve the throughput of the system. Several research works have been conducted to compare the system performance between NOMA and traditional orthogonal multiple access (OMA) [10]-[12]. In [10], the throughput maximization problem considering user fairness was addressed for both NOMA and OMA systems, and it proved that the proposed NOMA-based approach outperformed the OMAbased solution mathematically and numerically. Similar results for EE optimization problem were also observed in [11] for a downlink single input single output (SISO) NOMA system and in [13] for heterogeneous NOMA networks.

In addition to improving $\mathrm{SE}$ which is the motivation of NOMA, another key objective of future $5 \mathrm{G}$ networks is to maximize EE. The recent progress in the research on wireless power transfer (WPT) provides possibility of improving EE [14]. Furthermore, it is known that the radio frequency (RF) signals are the carriers of both information and energy, which makes it possible to combine WPT and wireless informa- 
tion transmission (WIT) in wireless communication systems. Motivated by this, an advanced technology named simultaneous wireless information and power transfer (SWIPT), has emerged recently in [15], aiming to prolong the battery-life of devices by achieving the parallel transmission of information and energy. As a result, SWIPT is regarded as a potential energy-efficient solution for the forthcoming 5G [14], which has attracted extensive attention in both academia and industry. In [15], Varshney defined a capacity-energy function considering that the terminals could simultaneously complete information decoding (ID) and energy harvesting (EH) without any limitation. Particularly, the capacity region for a SISO SWIPT system was studied. However, given that the receiver sensitivity for information and energy are fundamentally different, this capacity region cannot be achieved. Motivated by this, two practical receiving schemes have been proposed in [16] where ID and EH were separated through time domain and power domain respectively. These practical receiving schemes have promoted further development of SWIPT. In [17], Zhang and Ho considered a sum-rate maximization problem for both time switching (TS)-based and power splitting (PS)-based SWIPT multiple input multiple output (MIMO) broadcast systems and the optimal power transmission approach was developed. Under this setup, Song et al. in [18] further investigated the weighted mean squared error minimization problem. In [19], the weighted sum-rate maximization problem for a SWIPT multiple input single output (MISO) broadcast channel was studied, where the maximum transmit power constraint as well as the minimum harvested power constraint were considered. The work in [20] investigated the throughput maximization problem for an amplify-and-forward MIMO relay system by jointly optimizing the relay matrix and the precoder scheme. In particular, the sum-rate maximization problem was transformed to the mean square error minimization problem. In [21], the feasible rate-energy region was investigated for a SWIPT-based SISO OFDM system. The work in [22] studied SWIPT-based multi-user scenarios including TS-based time division multiple access (TDMA) systems as well as PSbased OFDMA systems. With the constraints of the minimum harvested power per-user and the maximum transmitted power, the optimal power allocation and the TS/PS ratio were investigated in order to obtain the maximum weighted sum-rate of the two systems. In [23], Shi et al. considered a quality of service (QoS)-based power minimization problem for a SWIPT-based multi-user MISO system by jointly optimizing the transmit beamforming and the PS factor. Besides, the harvested energy maximization problem with ID constraints was also studied in [24] for OFDM systems and in [25] for multiuser MISO systems. Furthermore, SWIPT enabled heterogeneous small cell network is regarded as a promising technique in the future mobile communication systems, and the resource allocation scheme of it was investigated in [26] and [27] so as to obtain the Nash equilibrium points. Most research works on SWIPT systems focused on maximizing sum rate or harvested energy. However, EE optimization for SWIPT systems has also been investigated recently [2], [28]. The work in [2] studied the optimal resource allocation for maximizing the EE of the OFDMA-based SWIPT system. Energy efficiency optimization with SWIPT in MIMO broadcast channels for internet of things (IoT) was studied in [28], where a practical linear power model taking into account the entire transmitreceive chain was proposed.

With the extensive fundamental studies on both SWIPT and NOMA, the combination of these two techniques has aroused great interest recently. In [29], authors studied SIC in a bipolar ad hoc network from a SWIPT point of view, and showed that SIC can significantly increase wireless power transfer without affecting the information decoding. The work in [30] considered both sum rate and data rate per-user optimization for the SWIPT-enabled NOMA systems, where two schemes for ID were proposed, namely "fixed decoding order" and "time sharing". It was demonstrated again that the system performance could be significantly improved through the integration of SWIPT and NOMA. Besides, both the downlink and the uplink of a wireless powered network, in the presence of interference, have been considered in [31]. Two different protocols were utilized for the downlink, i.e., NOMA and TDMA, while NOMA with time sharing was used for the uplink. By utilizing corresponding priority weights, authors fairly maximized the downlink/uplink users rates and showed that a relatively high downlink rate can be achieved. In [32], the secrecy sum-rate optimization problem for SWIPTenabled NOMA system has been investigated. Furthermore, cooperative SWIPT-based NOMA has also attracted great research interest. In [33], the application of SWIPT to NOMA networks in which users are spatially randomly located is investigated. A new co-operative SWIPT NOMA protocol is proposed, where three user selection schemes based on the user distances from the base station are proposed. The results confirm that the opportunistic use of node locations for user selection can achieve low outage probability and deliver superior throughput in comparison to the random selection scheme. In [34], authors studied the performance of a cell-edge user in a two-user MISO-NOMA system, where a cellcenter user acts as a relay to assist the cell-edge user and its relaying operation is powered by a hybrid TS/PS SWIPT protocol. The results demonstrate the achievable performance improvements in terms of outage performance of the proposed schemes in comparison to that of the OMA systems.

\section{A. Motivation and Contributions}

Since SWIPT and NOMA are two promising techniques for the forthcoming 5G, many researchers have investigated the performance of the integrated systems. The existing literatures indicate that SWIPT-enabled NOMA systems are able to provide performance improvement in many aspects compared to OMA systems, including energy harvesting [29], total throughput [30], user fairness [31], secrecy sum-rate [32], outage performance [33], [34], etc. To comply with the global commitments for handling the so-called resource crunch in a sustainable and economical manner, balancing the requirements of low power consumption and high data rata is significantly important. Hence, an alternative strategy is to consider the EE performance of the communication systems. On the other hand, the larger throughput is significantly important for the conventional cellular network, because the 
applications of mobile internet is becoming increasingly abundant, such as watching videos and playing online games, which indeed require relatively large data rates to avoid intermission. However, to support the functionality of the Internet of things and the massive machine-type communications scenarios, EE is important for small base stations with limited energy. In addition, it has been demonstrated that SWIPT is regarded as a potential energy-efficient solution for the forthcoming $5 \mathrm{G} / \mathrm{IoT}$, and the EE performance of the potential SWIPTenabled NOMA cellular networks remains unknown and it is thus valuable to be investigated.

Motivated by the aforementioned observations, we consider joint power allocation and TS control for QoS-constrained EE optimization in the downlink of SWIPT-enabled NOMA power-limited systems. The main contributions of this paper can be summarized as follows:

- The aim of this paper is to optimize the EE whilst satisfying the constraints on maximum transmit power budget, minimum data rate and minimum harvested energy per-terminal. The considered EE optimization problem is neither linear nor convex involving joint optimization of power allocation and TS factors in the presence of mutual interference, and thus is extremely difficult to solve directly. In order to tackle this, we develop a dual-layer iterative resource allocation algorithm where power allocation and TS control are separated for possible implementation in practical SWIPT-enabled NOMA systems. Specifically, the inner-layer is to optimize the power allocation whilst the outer-layer is used to control the TS assignment.

- In the inner-layer, the optimal power allocation was derived based on the Dinkelbach method, where the time switching factors are considered as constants. In particular, we have proved that the power allocation problem (under a fixed Dinkelbach parameter q) is convex, and hence it can be solved efficiently by applying the Lagrangian dual algorithm. In the outer-layer, Dinkelbach method is employed again to find out the optimal time switching factors with fix power allocation. In each iteration, the TS assignment problem is proved to be linear programming and hence the optimal solution can be achieved directly based on analytical property of linear function.

- A simplified but practical special case with equal TS factors is also considered. We have provide the computational complexity analysis for both the general case and the special case, where the computational complexity for the special case is remarkably lower.

- Numerical results demonstrate the EE performance of the proposed strategy of jointly optimizing the power allocation and the TS factors. More importantly, compared to the conventional OMA approach, our findings have illustrated that significant EE gain can be achieved by our proposed algorithm, and this has confirmed the advantages of integrating SWIPT into NOMA systems.

\section{B. Organization and Notation}

The remaining of this paper is structured as follows. The SWIPT-enabled NOMA system model and the corresponding EE maximization problem are presented in Section II. In Section III, a dual-layer algorithm is developed to find out the optimal joint power allocation and time switching assignment. A special case with equal time switching factor is further studied in Section IV. In Section V, the numerical results are presented to demonstrate the theoretical findings. Finally, we conclude this paper in Section VI.

The following notations are used throughout the paper. Non-bold and bold case letters represent scalar and vector respectively; $[x]^{+}$represents $\max (x, 0)$.

\section{System Model and Problem Formulation}

In this section, the SWIPT-enabled NOMA system model with TS-based terminals is presented first. Then we formulate the EE optimization problem mathematically.

\section{A. System Model}

As shown in Fig. 1, the considered SWIPT-enabled NOMA system with TS-based terminals includes one base station (BS) and $K$ different terminals; all are equipped with a single antenna. The total transmit power of the BS is limited to $P_{\max }$. We assume the power allocated to the $k^{t h}$ terminal in NOMA system is $P_{k}$. Furthermore, every terminal consists of an ID circuit and an EH rectification circuit. TS scheme is adopted to achieve ID and EH in two orthogonal time slots. For the $k^{t h}$ terminal, $\alpha_{k}$ denotes the portion of transmission time allocated to the ID time slot, and thus, $1-\alpha_{k}$ corresponds to that for the EH time slot.

Since all the terminals are sharing the same bandwidth $(B)$, they will interfere with each other when decoding information. Here SIC is applied to reduce mutual interference and improve the performance of the system. It has been shown in [35] that the capacity region of broadcast channels can be achieved when successive decoding in the order of increasing channel gains is applied. We denote the channel gains of the $k^{\text {th }}$ terminal as $h_{k}$, and let $g_{k}=\left|h_{k}\right|^{2}$. Without loss of generality, we assume that the channel gains satisfy the following condition: $g_{1} \leq g_{2} \leq \ldots \leq g_{K}$. In order to achieve the capacity boundary, the decoding order is set to $\{1,2, \ldots, K\}$ in which the terminal with the worst channel gain is decoded first. Channel state information (CSI) is assumed to be perfectly known at the BS and all the terminals. Note that the CSI at the receivers can be obtained from the channel estimation of the downlink pilots, while CSI at the transmitter can be acquired via uplink channel estimation in TDD mode.

Based on the system model presented above, the observation at the $k^{\text {th }}$ terminal is written as

$$
y_{k}=h_{k} \sum_{i=1}^{K} \sqrt{P_{i}} s_{i}+\omega_{k},
$$

where $s_{k}$ is the transmit signal for the $k^{t h}$ terminal, and $\omega_{k}$ is the additive Gaussian white noise (AWGN) with power $\sigma^{2}$. 


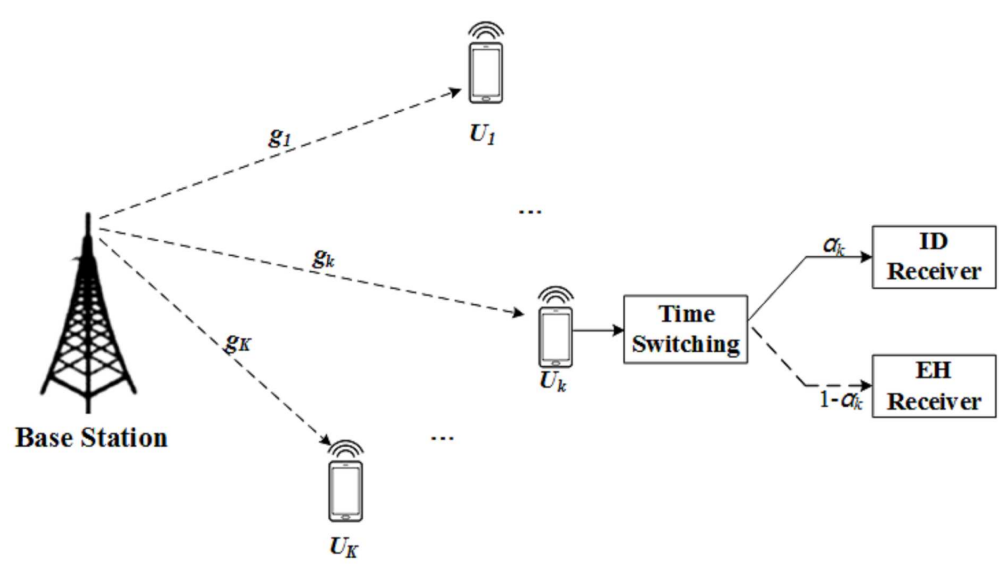

Fig. 1: A downlink SWIPT-enabled NOMA system with TS-based terminals.

Successive interference cancellation (SIC) will be carried out at the users. Therefore, the k-th user will detect the i-th user's message, $i<k$, and then remove the message from its observation, in a successive manner. The message for the $\mathrm{i}$-th user, $i>k$, will be treated as noise at the $\mathrm{k}$-th user. As a result, the data rate achievable to the $\mathrm{k}$-th user, $1 \leq k \leq(K-1)$, is given by

$$
R_{k}=\alpha_{k} B \log _{2}\left(1+\frac{g_{k} P_{k}}{\sigma^{2}+g_{k} \sum_{i=k+1}^{K} P_{i}}\right) .
$$

conditioned on $R_{j \rightarrow k} \geq \bar{R}_{j}$, where $\bar{R}_{j}$ denotes the targeted data rate of the j-th user, and $R_{j \rightarrow k}$ denotes the rate for the $\mathrm{k}$-th user to detect the $\mathrm{j}$-th users message, $j \leq k$. It should be noted that $\bar{R}_{k}$ is determined opportunistically by the users channel condition [4], i.e., $R_{j}>\bar{R}_{j}$, and hence it can be easily verified that the condition $R_{j \rightarrow k} \geq \bar{R}_{j}$ always holds since $g_{j} \leq g_{k}$ for $j<k$. Consequently the sum rate achieved by NOMA is given by

$$
\begin{gathered}
R_{\text {sum }}=\sum_{k=1}^{K-1} \alpha_{k} B \log _{2}\left(1+\frac{g_{k} P_{k}}{\sigma^{2}+g_{k} \sum_{i=k+1}^{K} P_{i}}\right) \\
+\alpha_{K} B \log _{2}\left(1+\frac{g_{K} P_{K}}{\sigma^{2}}\right) .
\end{gathered}
$$

It should also be noted that, for the case $\bar{R}_{k}$ is determined by the users QoS requirements, we should examine the probability of whether user can cancel others users messages or whether the system can ensure the users QoS requirements to be satisfied, and hence the outage performance is the primary target which is out of scope for this work.

On the other hand, the power harvested by the $k^{t h}$ terminal can be expressed as

$$
E_{k}=\left(1-\alpha_{k}\right) \eta g_{k} \sum_{i=1}^{K} P_{i}
$$

where $\eta$ is the power conversion efficiency of the EH receivers. The total harvested power of the system can thus be expressed as

$$
E_{\text {total }}=\sum_{k=1}^{K} E_{k}
$$

\section{B. Power Model}

Generally, the power consumption of the conventional wireless communication system is defined as the following linear model,

$$
P_{\text {total } 1}=\zeta \sum_{k=1}^{K} P_{k}+P_{C},
$$

where $\zeta$ accounts for the reciprocal of the power amplifier drain efficiency, and $P_{C}$ denotes the power consumed by the system hardware (circuit power).

Different from the conventional wireless communication system, the power consumption in a SWIPT system can be compensated by the transferred energy, i.e., harvested power at all the receivers. As a result, similar to the approach in [28], [36], the total power consumption of the mentioned system is given by

$$
P_{\text {total }}=\zeta \sum_{k=1}^{K} P_{k}+P_{C}-E_{\text {total }} .
$$

\section{Problem Formulation}

According to [37], the EE of the system can be defined as the ratio of the total achievable rates and the total power consumption of the communication system. Therefore, the EE of the considered SWIPT-enabled NOMA system with TSbased terminals can be formulated as follows

$$
\begin{aligned}
& \lambda_{E E} \triangleq \frac{R_{\text {total }}}{P_{\text {total }}} \\
= & \frac{\sum_{k=1}^{K} R_{k}}{\zeta \sum_{k=1}^{K} P_{k}+P_{C}-E_{\text {total }}} \\
= & \frac{B \sum_{k=1}^{K} \alpha_{k} \log _{2}\left(1+\frac{g_{k} P_{k}}{\sigma^{2}+g_{k} \sum_{i=k+1}^{K} P_{i}}\right)}{\zeta \sum_{k=1}^{K} P_{k}+P_{C}-\eta \sum_{k=1}^{K}\left(1-\alpha_{k}\right) g_{k} \sum_{i=1}^{K} P_{i}} .
\end{aligned}
$$

The objective of this work is to maximize the EE of the TS-based SWIPT-enabled NOMA system with the constraints of total transmitted power provided by BS as well as the quality of service (QoS) demanded by the terminals, including the minimum data rate constraint and the minimum harvested energy constraint per terminal. 
Hence, the EE optimization problem can be mathematically formulated as follows:

$$
\begin{aligned}
\max _{\alpha_{k}, P_{k}} & \frac{B \sum_{k=1}^{K} \alpha_{k} \log _{2}\left(1+\frac{g_{k} P_{k}}{\sigma^{2}+g_{k} \sum_{i=k+1}^{K} P_{i}}\right)}{\zeta \sum_{k=1}^{K} P_{k}+P_{C}-\eta \sum_{k=1}^{K}\left(1-\alpha_{k}\right) g_{k} \sum_{i=1}^{K} P_{i}} \\
\text { s.t. } \quad & \alpha_{k} B \log _{2}\left(1+\frac{g_{k} P_{k}}{\sigma^{2}+g_{k} \sum_{i=k+1}^{K} P_{i}}\right) \geq R_{\text {min }} \\
& \forall k \in \mathcal{K}, \\
& \left(1-\alpha_{k}\right) \eta g_{k} \sum_{i=1}^{K} P_{i} \geq E_{\text {min }}, \forall k \in \mathcal{K} \\
& \sum_{k=1}^{K} P_{k} \leq P_{\text {max }}, \\
& 0 \leq \alpha_{k} \leq 1, P_{k} \geq 0, \forall k \in \mathcal{K} .
\end{aligned}
$$

where $\mathcal{K}=\{1,2, \ldots, K\}$ denotes the set of all terminals. Equation (10) corresponds to the minimum rate constraint for each ID receiver while (11) corresponds to the minimum harvested power constraint for each EH receiver, in which $R_{\min }$ and $E_{\min }$ denote the minimum rate target and the minimum harvested power requirement respectively. In (12), the total power budget is set to $P_{\max }$. Besides, $0 \leq \alpha_{k} \leq 1$ and $P_{k} \geq 0$ for any $k \in \mathcal{K}$ are requested. To satisfy the minimum rate constraints per terminal (10), the system require a minimum transmit power denoted as $\bar{P}$. Similarly, the system require a minimum transmit power $\tilde{P}$ to meet the minimum harvested energy constraints per terminal (11). Since the total transmit power $\sum_{k=1}^{K} P_{k}$ should not exceed $P_{\text {max }}$, $\min \{\bar{P}, \tilde{P}\}>P_{\max }$ would lead to the infeasibility of this optimization problem.

We can observe that the objective function (9) is a rather complicated fraction, and thus the problem is a non-linear optimization problem. Besides, the problem involves two sets of variables, i.e., the time switching factor $\boldsymbol{\alpha}$ and transmitted power allocation $\boldsymbol{P}$, where $\boldsymbol{\alpha}=\left[\alpha_{1}, \alpha_{2}, \ldots, \alpha_{K}\right]$ and $\boldsymbol{P}=\left[P_{1}, P_{2}, \ldots, P_{K}\right]$. Jointly optimizing the two sets of variables makes the optimization problem (9)-(13) non-convex and therefore difficult to obtain the optimal solution directly. In the following sections, we will develop resource allocation schemes to jointly optimize $\boldsymbol{\alpha}$ and $\boldsymbol{P}$ in order to maximize EE. As stated in [38], for any optimization problems with multiple variables, we can analyze and solve the problem over some variables, regarding the rest as constants; and then solve the problem over the remaining variables. Therefore, we will separate $\boldsymbol{\alpha}$ and $\boldsymbol{P}$ when developing the optimization algorithm so as to overcome the difficulty. In other words, we firstly optimize $\boldsymbol{P}$ with a fixed $\boldsymbol{\alpha}$ (inner-layer process), and then turn to deal with $\alpha$ with the updated power allocation (outer-layer process).

\section{Joint Power Allocation and Time Switching CONTROL Algorithm}

In this section, we consider the joint power allocation and time switching control algorithm for the SWIPT-enabled NOMA system with multiple TS-based terminals. Note that the optimization problem in (9)-(13) belongs to a non-linear non-convex fractional programming problem, which involves two sets of variables and is rather difficult to obtain the solution directly. As a consequence, we develop a dual-layer iterative method where optimal power allocation $\boldsymbol{P}$ is obtained in the inner-layer process and the time switching factor $\boldsymbol{\alpha}$ is controlled in the outer-layer process. The proposed dual-layer iterative algorithm is shown as follows:

(i) Inner-layer process: to find out the optimal power allocation $\boldsymbol{P}$ of the optimization problem (9)-(13) under fixed time switching factor $\boldsymbol{\alpha}$;

(ii) Outer-layer process: to update the time switching factor $\boldsymbol{\alpha}$ with the power allocation $\boldsymbol{P}$ obtained in inner-layer process.

This process is repeated until convergence, i.e., there is no further improvement in EE.

\section{A. Power Allocation Approach under Fixed Time Switching Assignment}

In this section, the time switching factor $\alpha_{k}(\forall k \in \mathcal{K})$ is considered as a constant in $[0,1]$ so that the EE maximization problem (9)-(13) can be regarded as a power allocation problem. The fractional objective function makes the problem neither linear nor convex and thus difficult to solve straightforwardly. Since Dinkelbach method [39] has been widely applied to solve non-linear fractional optimization problem, we apply it to tackle our EE maximization problem. Particularly, we convert the fractional objective function into a subtractive form of numerator and denominator on the basis of the following proposition.

Proposition 1: For $U_{R}(\boldsymbol{P}) \geq 0$ and $U_{T}(\boldsymbol{P}) \geq 0$, the maximum achievable EE $q^{*}=\lambda_{E E}^{*}$ can be obtained supposing that

$$
\max _{\boldsymbol{P} \geq \mathbf{0}} U_{R}(\boldsymbol{P})-q^{*} U_{T}(\boldsymbol{P})=U_{R}\left(\boldsymbol{P}^{*}\right)-q^{*} U_{T}\left(\boldsymbol{P}^{*}\right)=0,
$$

where

$$
\begin{gathered}
U_{R}(\boldsymbol{P})=B \sum_{k=1}^{K} \alpha_{k} \log _{2}\left(1+\frac{g_{k} P_{k}}{\sigma^{2}+g_{k} \sum_{i=k+1}^{K} P_{i}}\right), \\
U_{T}(\boldsymbol{P})=\zeta \sum_{k=1}^{K} P_{k}+P_{C}-\eta \sum_{k=1}^{K}\left(1-\alpha_{k}\right) g_{k} \sum_{i=1}^{K} P_{i}, \\
q^{*}=\frac{U_{R}\left(\boldsymbol{P}^{*}\right)}{U_{T}\left(\boldsymbol{P}^{*}\right)} .
\end{gathered}
$$

Proof: Please refer to [39] for the proof of Proposition 1.

Proposition 1 provides a necessary and sufficient condition for developing the optimal power allocation approach. In general, an equivalent optimization problem with an objective function of subtractive form, such as $U_{R}(\boldsymbol{P})-q^{*} U_{T}(\boldsymbol{P})$, can be found to replace the original EE maximization problem with a fractional objective function, such that the solutions of the two optimization problems are equivalent. Furthermore, it is implied in [39] that the optimal solution can be obtained according to the equality condition in (14). Thus, rather than handling the original optimization problem (9)-(13) directly, 
we develop a power allocation algorithm for the equivalent optimization problem while meeting the condition in Proposition 1, which can be concluded in TABLE I.

It is necessary to prove the convergence of the Dinkelbach method-based algorithm in order to demonstrate its feasibility. Firstly, we prove that the EE (i.e., q) increases in each iteration. Then we prove that $q$ converges to the optimal value $q^{*}$ if the number of iterations is large enough. Note that $q^{*}$ meets the optimality condition in Proposition 1, i.e., $U_{R}\left(\boldsymbol{P}^{*}\right)-q^{*} U_{T}\left(\boldsymbol{P}^{*}\right)=F\left(q^{*}\right)=0$. Suppose that $\boldsymbol{P}^{(n)}$ is the optimal power allocation in the $n^{\text {th }}$ iteration, $q^{(n)} \neq q^{*}$ and $q^{(n+1)} \neq q^{*}$ denote the EE of the mentioned system in the $n^{\text {th }}$ and the $(n+1)^{t h}$ iteration respectively. It has been proved in [39] that $F\left(q^{(n)}\right)>0$ and $F\left(q^{(n+1)}\right)>0$ hold. On the other hand, for our proposed algorithm, $q^{(n+1)}=\frac{U_{R}\left(\boldsymbol{P}^{(n)}\right)}{U_{T}\left(\boldsymbol{P}^{(n)}\right)}$. Thus, we have the following expression

$$
\begin{aligned}
F\left(q^{(n)}\right) & =U_{R}\left(\boldsymbol{P}^{(n)}\right)-q^{(n)} U_{T}\left(\boldsymbol{P}^{(n)}\right) \\
& =q^{(n+1)} U_{T}\left(\boldsymbol{P}^{(n)}\right)-q^{(n)} U_{T}\left(\boldsymbol{P}^{(n)}\right) \\
& =\left(q^{(n+1)}-q^{(n)}\right) U_{T}\left(\boldsymbol{P}^{(n)}\right) .
\end{aligned}
$$

Since $U_{T}\left(\boldsymbol{P}^{(n)}\right)=\zeta \sum_{k=1}^{K} P_{k}^{(n)}+P_{C}-$ $\eta \sum_{k=1}^{K}\left(1-\alpha_{k}\right) g_{k} \sum_{i=1}^{K} P_{i}^{(n)}>0$ always holds in practice and $F\left(q^{(n)}\right)>0$, we can easily obtain $q^{(n+1)}>q^{(n)}$. Consequently, we can show that if the number of iterations $n$ is large enough, $q^{(n+1)} \rightarrow q^{(n)}$ and $F\left(q^{(n+1)}\right) \rightarrow 0$ hold, i.e., the optimality condition in Proposition 1 can be met.

As it can be seen from TABLE I, the crucial step for the developed Dinkelbach method-based algorithm is to deal with the following equivalent optimization problem with a given $q$ (step 3 in TABLE I)

$$
\begin{array}{rl}
\max _{P_{k} \geq 0} & B \sum_{k=1}^{K} \alpha_{k} \log _{2}\left(1+\frac{g_{k} P_{k}}{\sigma^{2}+g_{k} \sum_{i=k+1}^{K} P_{i}}\right) \\
& -q\left(\zeta \sum_{k=1}^{K} P_{k}+P_{C}-\eta \sum_{k=1}^{K}\left(1-\alpha_{k}\right) g_{k} \sum_{i=1}^{K} P_{i}\right) \\
\text { s.t. } \quad \alpha_{k} B \log _{2}\left(1+\frac{g_{k} P_{k}}{\sigma^{2}+g_{k} \sum_{i=k+1}^{K} P_{i}}\right) \geq R_{\text {min }} & \\
& \forall k \in \mathcal{K}, \\
& \left(1-\alpha_{k}\right) \eta g_{k} \sum_{i=1}^{K} P_{i} \geq E_{\min }, \forall k \in \mathcal{K} \\
& \sum_{k=1}^{K} P_{k} \leq P_{\max } .
\end{array}
$$

Proposition 2: For a fixed parameter $q$, the objective function (19) is strictly concave in $P_{k}(\forall k \in \mathcal{K})$.

Proof: See Appendix A.

Note that constraint (20) can be transformed as the following equivalent form

$$
\sigma^{2}+g_{k} \sum_{i=k}^{K} P_{i}-2^{\frac{R m i n}{\alpha_{k} B}}\left(\sigma^{2}+g_{k} \sum_{i=k+1}^{K} P_{i}\right) \geq 0, \forall k \in \mathcal{K},
$$

TABLE I: PROPOSED ITERATIVE POWER ALLOCATION ALGORITHM BASED ON DINKELBACH METHOD.

1) Let $n=0$ and $q^{(n)}=0$;

Set $\epsilon>0$ as the stopping criterion;

\section{2) REPEAT}

3) For a given $q^{(n)}$, solve (19)-(22) to obtain the power allocation $\boldsymbol{P}^{(n)}$;

4) IF $U_{R}\left(\boldsymbol{P}^{(n)}\right)-q^{(n)} U_{T}\left(\boldsymbol{P}^{(n)}\right) \leq \epsilon$

5) Convergence $=$ TRUE;

6) $\quad$ RETURN $\boldsymbol{P}^{*}=\boldsymbol{P}^{(n)}, q^{*}=q^{(n)}$;

7) ELSE

8) Convergence $=$ FALSE;

9) Set $n=n+1$ and $q^{(n)}=\frac{U_{R}\left(\boldsymbol{P}^{(n-1)}\right)}{U_{T}\left(\boldsymbol{P}^{(n-1)}\right)}$;

10) END IF

11) UNTIL Convergence $=$ TRUE.

which is clearly linear. Since constraints (21)-(22) are also linear and the objective function (19) is concave, the equivalent optimization problem based on Dinkelbach method (19)-(22) is a convex maximization problem, and zero duality gap exists. Lagrangian dual algorithm can be applied here to deal with the above convex optimization problem. The associated Lagrangian function of problem (19)-(22) can be given as

$$
\begin{aligned}
& \mathcal{L}(\boldsymbol{P}, \boldsymbol{\mu}, \boldsymbol{\nu}, \psi)=B \sum_{k=1}^{K} \alpha_{k} \log _{2}\left(1+\frac{g_{k} P_{k}}{\sigma^{2}+g_{k} \sum_{i=k+1}^{K} P_{i}}\right) \\
- & q\left(\zeta \sum_{k=1}^{K} P_{k}+P_{C}-\eta \sum_{k=1}^{K}\left(1-\alpha_{k}\right) g_{k} \sum_{i=1}^{K} P_{i}\right) \\
& -\sum_{k=1}^{K} \mu_{k}\left(R_{\text {min }}-\alpha_{k} B \log _{2}\left(1+\frac{g_{k} P_{k}}{\sigma^{2}+g_{k} \sum_{i=k+1}^{K} P_{i}}\right)\right) \\
& -\sum_{k=1}^{K} \nu_{k}\left(E_{\text {min }}-\left(1-\alpha_{k}\right) \eta g_{k} \sum_{i=1}^{K} P_{i}\right) \\
& -\psi\left(\sum_{k=1}^{K} P_{k}-P_{\text {max }}\right),
\end{aligned}
$$

where $\boldsymbol{\mu} \geq \mathbf{0}, \boldsymbol{\nu} \geq \mathbf{0}$ and $\psi \geq 0$ are the Lagrangian multipliers corresponding to the minimum rate constraints, the minimum harvested power constraints and the maximum transmitted power constraint, respectively. Thus, the dual objective function is given by

$$
g(\boldsymbol{\mu}, \boldsymbol{\nu}, \psi)=\max _{\boldsymbol{P}} \mathcal{L}(\boldsymbol{P}, \boldsymbol{\mu}, \boldsymbol{\nu}, \psi),
$$

and the dual optimization problem is formulated as follows

$$
\begin{array}{cl}
\min _{\boldsymbol{\mu}, \boldsymbol{\nu}, \psi} & g(\boldsymbol{\mu}, \boldsymbol{\nu}, \psi) \\
\text { s.t. } & \boldsymbol{\mu} \geq \mathbf{0}, \boldsymbol{\nu} \geq \mathbf{0} \text { and } \psi \geq 0 .
\end{array}
$$

In this work, we firstly develop an iterative scheme based on gradient descent algorithm so as to obtain the optimal 
$\boldsymbol{P}$ for the dual optimization problem (26). Particularly, $P_{k}$ can be updated successively according to the gradient of the Lagrangian function (24) with respect to $P_{k}$, which is formulated as follows

$$
\begin{aligned}
& \nabla_{P_{k}} \mathcal{L} \\
& :=\frac{B}{\ln 2} \cdot\left(\frac{\alpha_{1} g_{1}}{\sigma^{2}+g_{1} \theta_{1}}+\sum_{i=2}^{k}\left(\frac{\alpha_{i} g_{i}}{\sigma^{2}+g_{i} \theta_{i}}-\frac{\alpha_{i-1} g_{i-1}}{\sigma^{2}+g_{i-1} \theta_{i}}\right)\right) \\
& +\frac{B}{\ln 2} \cdot\left(\frac{\mu_{1} \alpha_{1} g_{1}}{\sigma^{2}+g_{1} \theta_{1}}+\sum_{i=2}^{k}\left(\frac{\mu_{i} \alpha_{i} g_{i}}{\sigma^{2}+g_{i} \theta_{i}}-\frac{\mu_{i-1} \alpha_{i-1} g_{i-1}}{\sigma^{2}+g_{i-1} \theta_{i}}\right)\right) \\
& -q\left(\zeta-\eta \sum_{i=1}^{K}\left(1-\alpha_{i}\right) g_{i}\right)+\eta \sum_{i=1}^{K} \nu_{i}\left(1-\alpha_{i}\right) g_{i}-\psi, \\
& P_{k}^{(n)}=\left[P_{k}^{(n-1)}+t \nabla_{P_{k}}^{(n-1)} \mathcal{L}\right]^{+},
\end{aligned}
$$

where $\theta_{i}=\sum_{j=i}^{K} P_{j}(\forall i \in \mathcal{K})$ and particularly $\theta_{K+1}=0$; $t$ denotes the iteration step size of $P_{k} ; P_{k}^{(n)}$ and $P_{k}^{(n-1)}$ represent the power allocation of the $n^{t h}$ and the $(n-1)^{t h}$ iteration respectively.

After we find out the optimal $\boldsymbol{P}$, now we turn to cope with the dual optimization problem (26) so as to achieve the optimal Lagrangian multipliers $\boldsymbol{\mu} \geq \mathbf{0}, \boldsymbol{\nu} \geq \mathbf{0}$ and $\psi \geq 0$. Note that the objective function as well as all the constraints of the dual problem are all linear with respect to Lagrangian multipliers. Thus, the dual problem is convex over the dual variables $\boldsymbol{\mu}, \boldsymbol{\nu}, \psi$ which can be optimized through a onedimensional searching algorithm. Nevertheless, the gradient algorithm is not necessarily feasible owing to the fact that the dual function (25) is not guaranteed to be differentiable. Here we employ the well-known sub-gradient algorithm to update the dual variables $\boldsymbol{\mu}, \boldsymbol{\nu}, \psi$, and the sub-gradient direction is presented in the following Lemma.

Lemma 1. The subgradient of the dual function $g(\boldsymbol{\mu}, \boldsymbol{\nu}, \psi)$ is denoted as follows

$$
\begin{gathered}
\nabla_{\mu_{k}} g:=\alpha_{k} B \log _{2}\left(1+\frac{g_{k} P_{k}}{\sigma^{2}+g_{k} \sum_{i=k+1}^{K} P_{i}}\right)-R_{\text {min }}, \\
\forall k \in \mathcal{K}, \\
\nabla_{\nu_{k}} g:=\left(1-\alpha_{k}\right) \eta g_{k} \sum_{i=1}^{K} P_{i}-E_{\text {min }}, \quad \forall k \in \mathcal{K}, \\
\nabla_{\psi} g:=P_{\text {max }}-\sum_{k=1}^{K} P_{k} .
\end{gathered}
$$

Proof: Please refer to [40] for a proof of Lemma 1.

Therefore, the dual variables can be updated according to the following expression

$$
\begin{gathered}
\mu_{k}^{(m)}=\left[\mu_{k}^{(m-1)}+\omega^{(m-1)} \nabla_{\mu_{k}} g\right]^{+}, \forall k \in \mathcal{K}, \\
\nu_{k}(m)=\left[\nu_{k}^{(m-1)}+\omega^{(m-1)} \nabla_{\nu_{k}} g\right]^{+}, \forall k \in \mathcal{K}, \\
\psi^{(m)}=\left[\psi^{(m-1)}+\omega^{(m-1)} \nabla_{\psi} g\right]^{+}
\end{gathered}
$$

TABLE II: PROPOSED ITERATIVE POWER ALLCATION SCHEME BASED ON LAGRANGE DUAL THEORY.

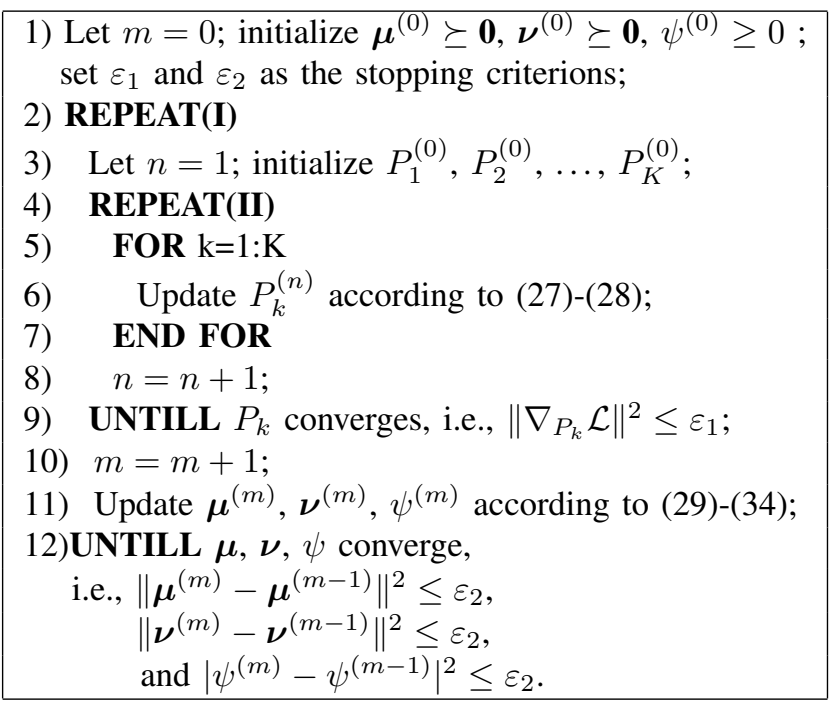

where $\omega$ denotes the iteration step size of dual variables.

Now we can conclude the approach to cope with the optimization problem in (19)-(22) under a given parameter $q$ in each iteration, which is presented in TABLE II.

\section{B. Time Switching Control Scheme under Fixed Power Allo- cation}

In the previous section, we have developed a Dinkelbach method-based iterative approach to obtain the optimal power allocation $\boldsymbol{P}$ under a fixed time switching assignment. However, the achieved EE $\lambda_{E E}$ is not necessarily optimal since the time switching factor $\boldsymbol{\alpha}$ is regarded as a constant vector and not guaranteed to be optimal. Therefore, in this section, we will develop a scheme to optimize $\boldsymbol{\alpha}$ under fixed $\boldsymbol{P}$.

With a fixed power allocation $\boldsymbol{P}$ achieved in the innerlayer process, the original optimization problem in (9)-(13) is accordingly simplified as follows

$$
\begin{aligned}
\max _{0 \leq \alpha_{k} \leq 1} & \frac{\sum_{k=1}^{K} \mathcal{A}_{k} \alpha_{k}}{\mathcal{B}+\sum_{k=1}^{K} \mathcal{C}_{k} \alpha_{k}} \\
\text { s.t. } & \mathcal{A}_{k} \alpha_{k} \geq R_{\text {min }}, \quad \forall k \in \mathcal{K}, \\
& \mathcal{C}_{k}\left(1-\alpha_{k}\right) \geq E_{\text {min }}, \forall k \in \mathcal{K},
\end{aligned}
$$

where we denote

$$
\begin{gathered}
\mathcal{A}_{k}=B \log _{2}\left(1+\frac{g_{k} P_{k}}{\sigma^{2}+g_{k} \sum_{i=k+1}^{K} P_{i}}\right) \geq R_{\text {min }}, \forall k \in \mathcal{K} \\
\mathcal{B}=\zeta \sum_{k=1}^{K} P_{k}+P_{C}-\eta \sum_{k=1}^{K} g_{k} \sum_{i=1}^{K} P_{i} \\
\mathcal{C}_{k}=\eta g_{k} \sum_{i=1}^{K} P_{i}, \forall k \in \mathcal{K}
\end{gathered}
$$

According to (36) and (37), we have $\alpha_{k} \geq R_{\min } / \mathcal{A}_{k}$ and $\alpha_{k} \leq 1-E_{\min } / \mathcal{C}_{k}$. Therefore, there is no feasible solution if 
$R_{\text {min }} / \mathcal{A}_{k}>1-E_{\min } / \mathcal{C}_{k}$. Here Dinkelbach method is employed again to cope with the fractional programming problem in (35)-(37), and the corresponding Dinkelbach method-based problem is given by

$$
\begin{array}{cl}
\max _{0 \leq \alpha_{k} \leq 1} & \Lambda_{E E}(\boldsymbol{\alpha}) \triangleq \sum_{k=1}^{K} \mathcal{A}_{k} \alpha_{k}-\beta\left(\mathcal{B}+\sum_{k=1}^{K} \mathcal{C}_{k} \alpha_{k}\right) \\
\text { s.t. } & \mathcal{A}_{k} \alpha_{k} \geq R_{\text {min }}, \forall k \in \mathcal{K} \\
& \mathcal{C}_{k}\left(1-\alpha_{k}\right) \geq E_{\text {min }}, \forall k \in \mathcal{K} .
\end{array}
$$

Thus, (35)-(37) is converted into a linear programming problem, in which the optimal time switiching factor $\boldsymbol{\alpha}$ can be achieved according to the following Proposition.

Proposition 3: With a fixed power allocation $\boldsymbol{P}$ and $a$ given parameter $\beta$, the optimal time switiching factor can be calculated as

$$
\alpha_{k}^{*}=\left\{\begin{array}{ll}
\max \left\{0,1-\frac{E_{\min }}{\mathcal{C}_{k}}\right\}, & \text { if } \mathcal{A}_{k}-\beta \mathcal{C}_{k} \geq 0 \\
\min \left\{1, \frac{R_{\min }}{\mathcal{A}_{k}}\right\}, & \text { if } \mathcal{A}_{k}-\beta \mathcal{C}_{k}<0
\end{array},\right. \text {, }
$$

where $\mathcal{A}_{k}-\beta \mathcal{C}_{k}$ is the first-order derivative of $\Lambda_{E E}(\boldsymbol{\alpha})$ with respect to $\alpha_{k}$, i.e., $\frac{\partial \Lambda_{E E}(\boldsymbol{\alpha})}{\partial \alpha_{k}}$.

Proof: The proof of Proposition 3 is not difficult to obtain according to the analytical property of linear function, and hence is omitted for brevity.

Similar to the previous, we update the Dinkelbach parameter $\beta$ with the following expression

$$
\beta^{*}=\frac{\sum_{k=1}^{K} \mathcal{A}_{k} \alpha_{k}^{*}}{\mathcal{B}+\sum_{k=1}^{K} \mathcal{C}_{k} \alpha_{k}^{*}},
$$

and then continue to deal with the optimization problem in (41)-(43), which is repeated until convergence, i.e.,

$$
\begin{aligned}
& \max _{0 \leq \alpha_{k} \leq 1} \sum_{k=1}^{K} \mathcal{A}_{k} \alpha_{k}-\beta^{*}\left(\mathcal{B}+\sum_{k=1}^{K} \mathcal{C}_{k} \alpha_{k}\right) \\
= & \mathcal{A}_{k} \alpha_{k}^{*}-\beta^{*}\left(\mathcal{B}+\sum_{k=1}^{K} \mathcal{C}_{k} \alpha_{k}^{*}\right)=0 .
\end{aligned}
$$

Note that the EE $\beta$ increases in each iteration and the proof of the convergence is similar to the previous Dinkelbach method-based algorithm, and hence its feasibility can be guaranteed.

\section{Joint Power Allocation and Time Switching Solution}

The joint power allocation and time switching strategy for the original optimization problem (9)-(13) thus is summarized as follows

$$
\begin{aligned}
& \underbrace{\boldsymbol{P}[0], \boldsymbol{\alpha}[0] \rightarrow \lambda_{E E}[0]}_{\text {Initialization }} \rightarrow \cdots \rightarrow \underbrace{\boldsymbol{P}[t] \rightarrow \boldsymbol{\alpha}[t], \lambda_{E E}[t]}_{t^{t h} \text { iteration }} \\
& \rightarrow \underbrace{\boldsymbol{P}[t+1] \rightarrow \boldsymbol{\alpha}[t+1], \lambda_{E E}[t+1]}_{(t+1)^{t h} \text { iteration }} \rightarrow \cdots,
\end{aligned}
$$

where $\boldsymbol{P}[t], \boldsymbol{\alpha}[t]$ and $\lambda_{E E}[t]$ are the power allocation, the time switching assignment and the obtained energy efficiency in the $t^{\text {th }}$ iteration, respectively. With the developed power allocation algorithm and time switching control strategy, the
TABLE III: THE COMPLETE SOLUTION TO THE EE OPTIMIZATION PROBLEM FOR THE SWIPT-ENABLED NOMA SYSTEM.

1) Initialize $\boldsymbol{P}[0], \boldsymbol{\alpha}[0]$ and calculate $\lambda_{E E}[0]$;
set a small $\xi$ as the stopping criterion; $t=0$;
2) REPEAT
3) $\quad t=t+1$;
4) Let $q^{(0)}[t]=\lambda_{E E}[t-1]$, solve the optimization
problem $(19)$-(22) under the fixed TS assignment
$\boldsymbol{\alpha}[t-1]$ according to TABLE I and II;
obtain the corresponding $\boldsymbol{P}[t]$ and $q^{*}[t]$
5)
Let $\beta^{(0)}[t]=q^{*}[t]$, solve the optimization problem
(41)-(43) under the fixed power allocation $\boldsymbol{P}[t]$
according to the analysis in (44)-(46);
obtain the corresponding $\boldsymbol{\alpha}[t]$ and $\beta[t] ;$
6) $\lambda_{E E}[t]=\beta[t]$;
7) UNTIL converge, i.e., $\left|\lambda_{E E}[t]-\lambda_{E E}[t-1]\right|^{2} \leq \xi$.

update process is repeated until no further improvement in EE can be obtained, i.e., $\lambda_{E E}[t+1]=\lambda_{E E}[t]$. To summarize, the complete solution to the EE optimization problem in (9)(13) for the SWIPT-enabled NOMA system with TS-based terminals is concluded in TABLE III. We can also prove that the energy efficiency $\lambda_{E E}$ increases in each iteration shown in TABLE III and the convergence of the complete algorithm exists.

We provide a complexity analysis for the proposed duallayer iterative process. In SIC, we successively cancel the interference of each terminal so that the computational complexity depends on the number of terminals, which is approximately of order $\mathcal{O}\left(K^{2.376}\right)$ [41]. Moreover, the computational complexity for the Dinkelbach method-based algorithm with stopping criteria $\epsilon$ is $\mathcal{O}\left(\frac{1}{\epsilon^{2}} \log (K)\right)$ [39]. Note that Dinkelbach method is employed both in the inner-layer and the outerlayer iterative process. Hence, the computational complexity of the proposed dual-layer iterative algorithm is approximately of order $\mathcal{O}\left(\frac{1}{\epsilon_{1}^{2}} \frac{1}{\epsilon_{2}^{2}}(\log (K))^{2} K^{2.376}\right)$, assuming that $\epsilon_{1}$ and $\epsilon_{2}$ are the stopping criteria in the inner-layer and the outer-layer iterative process respectively.

\section{Special Case with Equal Time Switching FACTORS}

In the previous section, we focus on the case that the terminals are equipped with different SWIPT-receivers, and accordingly the optimal time switching factors are usually different among terminals, which causes that the proposed joint resource allocation algorithm is extremely complex. In this section, we consider a special case where $\alpha_{1}=\alpha_{2}=$ $\ldots=\alpha_{K}=\alpha$, and then propose an efficient algorithm for jointly optimizing the power allocation and the time switching assignment.

Note that Proposition 2 still holds when supposing $\alpha_{1}=$ $\alpha_{2}=\ldots=\alpha_{K}=\alpha$, and the power allocation approach under fixed time switching assignment proposed in the previous section is also appliable in this case. However, the 
time switching control scheme under fixed power allocation can be further simplified. With equal time switching factors $\alpha_{1}=\alpha_{2}=\ldots=\alpha_{K}=\alpha$ and a given power allocation $\boldsymbol{P}$, the optimization problem (9)-(13) can be rewritten as follows

$$
\begin{aligned}
\max _{0 \leq \alpha \leq 1} & \frac{\alpha B \sum_{k=1}^{K} \log _{2}\left(1+\frac{g_{k} P_{k}}{\sigma^{2}+g_{k} \sum_{i=k+1}^{K} P_{i}}\right)}{\zeta \sum_{k=1}^{K} P_{k}+P_{C}-(1-\alpha) \eta \sum_{k=1}^{K} g_{k} \sum_{i=1}^{K} P_{i}} \\
\text { s.t. } & \alpha B \log _{2}\left(1+\frac{g_{k} P_{k}}{\sigma^{2}+g_{k} \sum_{i=k+1}^{K} P_{i}}\right) \geq R_{\text {min }} \\
& \forall k \in \mathcal{K} \\
& (1-\alpha) \eta g_{k} \sum_{i=1}^{K} P_{i} \geq E_{\text {min }}, \forall k \in \mathcal{K}
\end{aligned}
$$

Proposition 4: The objective function in (48) is monotonically increasing with respect to $\alpha$.

Proof: See Appendix B.

According to the constraints in (49) and (50), we have

$$
\alpha \geq \max _{k}\left\{\frac{R_{\text {min }}}{B \log _{2}\left(1+\frac{g_{k} P_{k}}{\sigma^{2}+g_{k} \sum_{i=k+1}^{K} P_{i}}\right)}\right\} \triangleq \alpha_{\text {min }},
$$

and

$$
\alpha \leq \min _{k}\left\{1-\frac{E_{\min }}{\eta g_{k} \sum_{i=1}^{K} P_{i}}\right\}=1-\frac{E_{\text {min }}}{\eta g_{1} \sum_{i=1}^{K} P_{i}} \triangleq \alpha_{\max } .
$$

There is no feasible solution to the optimization problem (48)-(50) if $\alpha_{\min }>\alpha_{\max }$; otherwise, the optimal time switching factor for the problem (48)-(50) can be formulated according to Proposition 4 as

$$
\alpha^{*}=1-\frac{E_{\min }}{\eta g_{1} \sum_{i=1}^{K} P_{i}} .
$$

\section{A. Computational Complexity Analysis}

In this subsection, we analyze the computational complexity of the proposed joint optimization algorithms for both the general case and the special case, which are the key components of our dual-layer resource allocation framework.

1) Complexity analysis for the general case: The interference of each terminal is successively cancelled in SIC process, and hence the computational complexity depends on the number of terminals which is approximately of order $\mathcal{O}\left(K^{2.376}\right)$ [41]. In the inner-layer process, the computational complexity for Dinkelbach method with stopping criteria $\epsilon$ is $\mathcal{O}\left(\frac{1}{\epsilon^{2}} \log (K)\right)$ [39]. Furthermore, each iteration in the proposed Dinkelbach method can be regarded as a convex optimization problem, and thus the computational complexity for solving this problem is $\mathcal{O}(\log (1 / \varepsilon))$ [42], where $\varepsilon$ is the error tolerance for algorithm termination. In the outer-layer process, the Dinkelbach method is adopted again with the complexity of $\mathcal{O}\left(\frac{1}{\epsilon^{2}} \log (K)\right)$. Each iteration is required to compute the fist order derivative of $\Lambda_{E E}(\boldsymbol{\alpha})$ with respect to $\alpha_{k}$, i.e., $\frac{\partial \Lambda_{E E}(\boldsymbol{\alpha})}{\partial \alpha_{k}}$, and thus has a complexity of $\mathcal{O}(K)$. To sum up, the total computational complexity for the proposed dual-layer process is approximately $\mathcal{O}\left(\frac{1}{\epsilon^{4}} \log \left(\frac{1}{\varepsilon}\right) K^{3.376}(\log (K))^{2}\right)$.

2) Complexity analysis for the special case: The difference of the complexity between the general and the spe-

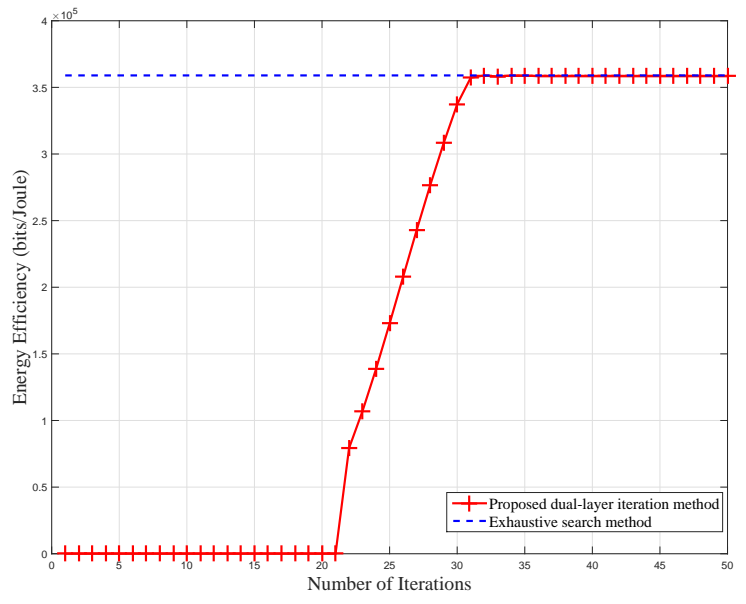

Fig. 2: Convergence performance of the proposed joint power allocation and time switching assignment algorithm.

cial case lies in the outer-layer. In particular, the calculation for the first order derivative with respect to TS factors is not needed and thus the computational complexity for the proposed dual-layer approach can be further reduced to $\mathcal{O}\left(\frac{1}{\epsilon^{4}}(\log (K))^{2} \log \left(\frac{1}{\varepsilon}\right) K^{2.376}\right)$.

\section{Simulation Results}

In this section, numerical results are presented to demonstrate the performance of the theoretical findings and the superiority of the proposed algorithms in terms of EE. The distance-dependent path-loss is given by $128.1+37.6 \log _{10} d$ [43], in which $d$ is the distance between the terminal receiver and the transmitter in $\mathrm{km}$. Log-Normal shadowing with standard deviation of $8 \mathrm{~dB}$ is considered. Besides, for the small-scale fading, each terminal experiences independent Rayleigh fading with unit variance. In other words, the smallscale fading is an independent and identically distributed zero mean circularly symmetric complex Gaussian (ZMCSCG) random variable with variance of 1 . In particular, all terminals are considered to be randomly located within $20 \mathrm{~m}$ to the corresponding BS and all the results are averaged over various random locations of terminals. In our simulations, the channel noise is considered as AWGN with power $\sigma^{2}=0.001 \mathrm{~W}$; the drain efficiency of the power amplifier $\zeta$ is set to $38 \%$ whereas the EH efficiency is taken to be $\eta=10 \%$; the bandwidth of the system is set to $B=1 M H z$ [44]. In addition, the static circuit power at the transmitter $P_{C}$ is assumed to be $5 W$. It should be noted that these system parameters are merely chosen to demonstrate the EE performance in an example and can easily be modified to any other values depending on the specific scenario under consideration.

In the first simulation, the convergence performance of the proposed joint power allocation and time switching assignment algorithm is studied. We take the case of three terminals for example. Besides, we set $P_{\max }=20 \mathrm{~W}, R_{\min }=2 \mathrm{Mbits} / \mathrm{s}$ and $E_{\min }=0.2 W$. The $\mathrm{EE}$ achieved by the proposed joint resource allocation scheme is compared with the EE performance obtained through the exhaustive search method. 


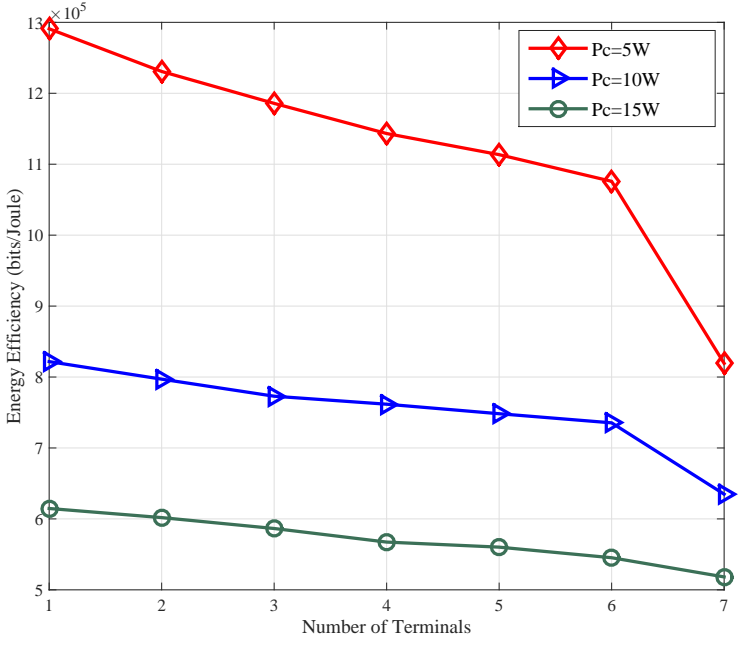

Fig. 3: The performance of the proposed algorithm with different number of terminals and $P_{C}$ (EE vs number of terminals and $P_{C}$ ).

As shown in Fig. 2, it can be observed that the EE converges to a stable value (optimal EE based on exhaustive searching). This result is in line with our theoretical analysis where the proposed joint scheme is efficient compared to the exhaustive search method for obtaining the optimal power allocation as well as the optimal time switching factor.

Then we investigate the EE performance of the proposed algorithm with different number of terminals as well as different circuit power $P_{C}$. In this simulation, the parameters of constraints are similar to the previous. The number of terminals varies from 1 to 7 whereas $P_{C}$ is set to $5 \mathrm{~W}, 10 \mathrm{~W}$ and $15 \mathrm{~W}$. From Fig. 3, it is obvious that EE decreases as $P_{C}$ increases. This is because the increasing circuit power directly increases the total power consumption without having any further impact on ID or EH. On the other hand, for a fixed circuit power $P_{C}$, we can draw a conclusion that $\mathrm{EE}$ is nonincreasing with respect to the number of terminals. In fact, the non-orthogonality of the channel access is the main reason for this observation. In NOMA systems, the inter-user interference would enhance with the increasing number of terminals. This reveals that a considerably higher transmit power is needed so as to achieve the minimum rate requirement for each terminal. On the other hand, the power conversion efficiency of the EH receiver $\eta$ is usually not very high (e.g. $\eta=10 \%$ ) due to the limitation of the practical hardware. Consequently, the increase in total transmit power is much larger than the total harvested power when the number of terminals is large, resulting in a significant increase in the actual power consumption and hence a poor EE performance. Besides, a big drop in EE occurs when the number of terminals exceeds six. This is because the considered power-limited system is most likely to fail to meet the QoS requirements of all terminals, and the corresponding EE is set to zero in this case. Therefore, this leads to a big drop in the EE averaged over randomly generated channels.

In the next simulation, the EE performance of the proposed joint power allocation and time switching assignment algorithm under various constraints are evaluated and presented

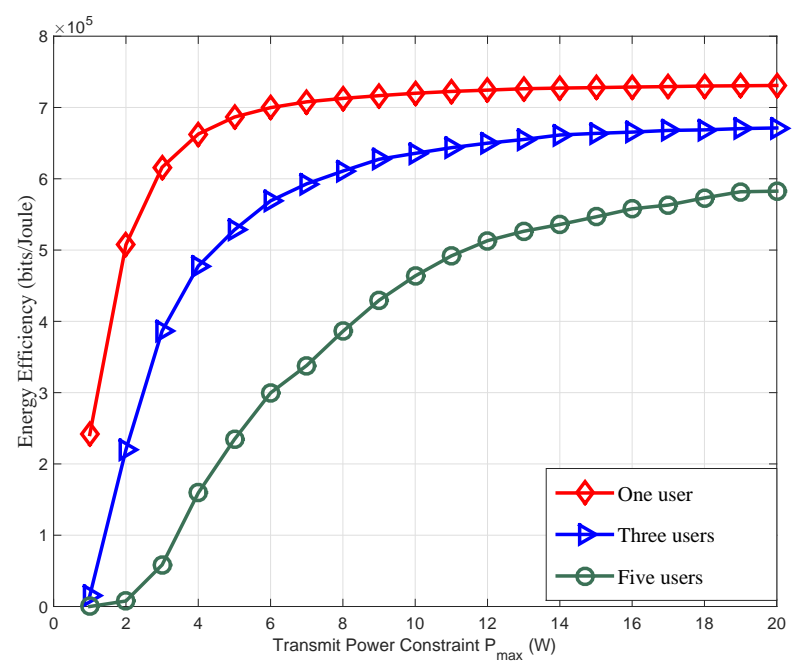

Fig. 4: The performance of the proposed algorithm with different power budget constraints (EE vs transmitted power budget).

in Fig. 4, Fig. 5 and Fig. 6. The circuit power is fixed to $P_{C}=5 \mathrm{~W}$ and the number of terminals is set to 1,3 and 5 for comparison. We first investigate the EE performance of the proposed solutions with different power budgets $P_{\max }$. In particular, we assume that the power budget varies within a range of $1 W \leq P_{\max } \leq 20 \mathrm{~W}$, and the constraints of the minimum rate and the minimum harvested power are set to $R_{\text {min }}=2 \mathrm{Mbits} / \mathrm{s}$ and $E_{\min }=0.2 \mathrm{~W}$ respectively. As shown in Fig. 4, the optimal EE achieved by the proposed scheme are monotonically non-decreasing in the total transmit power constraint. Particularly, the EE increases dramatically with a smaller power budget, and then approaches an asymptotic value where a balance among the achievable rates and the total power consumption is obtained. In other words, the additional power budget does not constitute to extra gain in EE, and similar results are observed in the conventional single-cell systems [45], multi-cell systems [46], and two-tier HetNets [47]. Besides, if there are more terminals in the network, the power budget $P_{\max }$ to reach the stable EE is required to be higher. These two results make sense when we expect to achieve better EE performance with a limited transmit power. We next show in Fig. 5 the maximum EE as well as the optimal TS ratio under different harvested power requirements. We set the harvested power constraint within the range of $0.2 W$ to $2 W$, and the constraints of power budget and the minimum rate are set to $P_{\max }=20 \mathrm{~W}$ and $R_{\min }=2 \mathrm{Mbits} / \mathrm{s}$ respectively. It can be seen in Fig. 5 that the maximum EE as well as the optimal TS ratio drops as the demanded harvested power increases. To satisfy a higher harvested energy demand, a higher ratio of transmit time for $\mathrm{EH}$ is required, which leads to a decline in $\alpha^{*}$. Moreover, extra transmitted power is needed to guarantee the quality of data rate, which leads to an imbalance between the numerator (transmission rate) and the denominator (power consumption) of the EE metric. Note that $\alpha^{*}=0$ means that the system fails to satify the QoS of the all terminals, and hence the energy efficiency $\lambda_{E E}=0$. At last, the maximum EE as well as the optimal TS ratio 


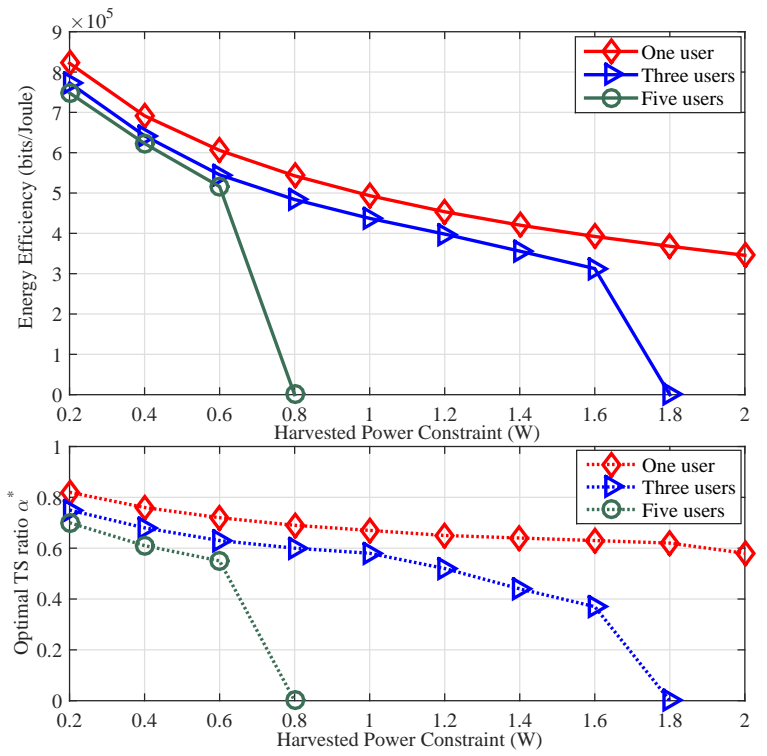

Fig. 5: The performance of the proposed algorithm with different harvested power constraints (EE vs harvested power constraint).

under different minimum rate constraints is presented in Fig. 6. Similarly, an increase in optimal TS ratio is required to achieve a high data rate demand, and thus more transmitted power is necessary so as to guarantee the quality of harvested power. Therefore, a similar trend can also be seen for the case of varying the minimum rate constraint, where the optimal EE achieved by the proposed approach remains unchanged up to a certain minimum transmission rate requirement, but decreases thereafter. Furthermore, we can conclude from Fig. 4 to Fig. 6 that $\mathrm{EE}$ is always decreasing with increasing number of terminals, regardless of the constraints of minimum rate, minimum harvester energy or maximum transmission power. This phenomenon is in consistence of the result in Fig. 3, and the reason for this has been discussed.

In the last simulation, we compare the EE performance among different approaches. Without loss of generality, we analyse the case where there are three terminals in the network. The curve marked as "Proposed SWIPT-enabled NOMA EEopt" denotes the EE performance of the proposed joint power allocation and time switching control scheme for SWIPTenabled NOMA system. The curve "SWIPT-enabled NOMA Rate-opt" represents the sum rate maximization scheme in the considered SWIPT-enabled NOMA system, where the objective function has been changed to maximizing the sum rate. It can be observed that with the increasing total transmit power budget $P_{\max }$, the EE achieved by "SWIPT-enabled NOMA Rate-opt" first increases and then decreases with $P_{\max }$. That is because for the rate maximization approach, the whole transmit power will be allocated for all the terminals to obtain the maximum data rate. However, when the transmit power is increased to a certain extent, the effectiveness on the improvement of data rate is not significant due to the property of $\log$ function. Therefore, the achieved EE drops after certain power level. On the other hand, since the additional power

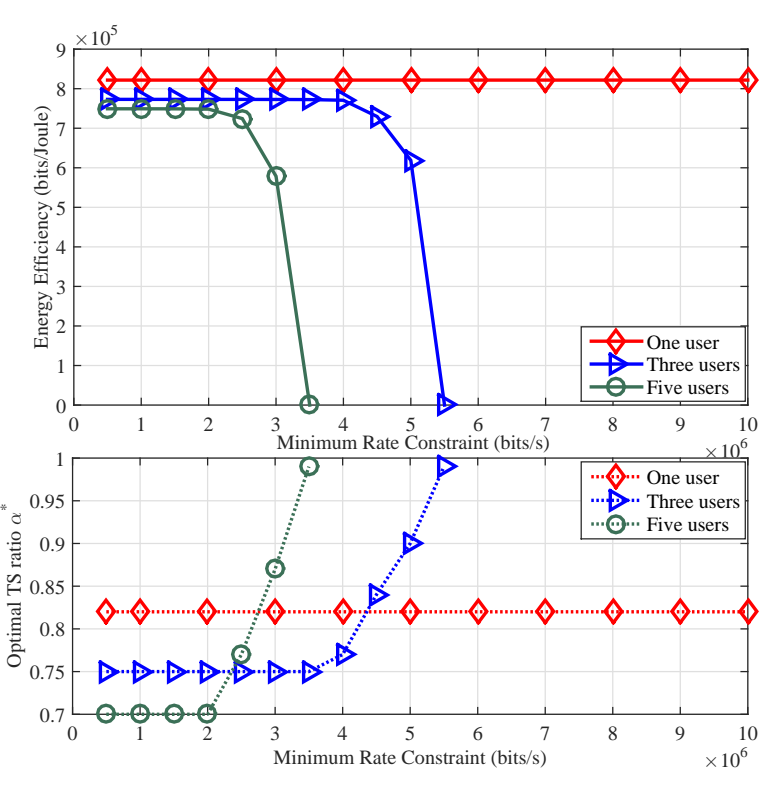

Fig. 6: The performance of the proposed algorithm with different minimum rate constraints (EE vs minimum rate constraint).

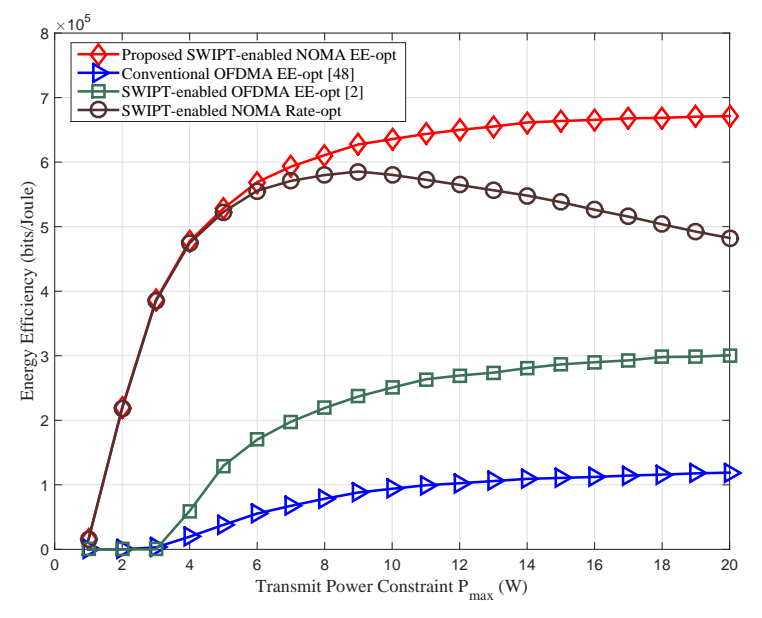

Fig. 7: Impact of the maximum transmit power on the EE performance under different resource allocation approaches.

budget does not contribute to extra gain in EE, the EE achieved by the proposed joint power allocation and time switching control algorithm is monotonically non-decreasing in the total transmit power constraint $P_{\max }$ (similar to the results in Fig. 4). In addition, the EE performance of OMA system is also presented for comparison. In particular, we take into account the approach that maximize the EE in SWIPT-enabled OFDMA system [2] and the approach that maximize the EE in a conventional OFDMA system [48], which are marked as "SWIPT-enabled OFDMA EE-opt [2]" and "conventional OFDMA EE-opt [48]" respectively. It is clear that the EE achieved by the proposed SWIPT-NOMA scheme outperforms both the SWIPT-OFDMA scheme and the conventional OFDMA-based scheme, which has demonstrated the applicability of our proposed approach and confirmed the advantages of integrating SWIPT into NOMA system. 


\section{CONCLUSIONS}

In this paper, we have addressed the $\mathrm{EE}$ optimization problem for a SWIPT-enabled NOMA system with TS-based terminals. Our goal is to maximize the EE whilst satisfying certain constraints in terms of maximum transmitted power budget, minimum rate and minimum harvested power. The corresponding EE optimization problem, which involves joint optimization of the TS factors and power allocation, is nonlinear and non-convex and thus the solution is non-trivial and cannot be derived directly. In order to obtain a feasible solution for this problem, we develop a dual-layer iterative resource allocation algorithm to tackle the multivariable optimization problem. In particular, Lagrangian dual algorithm for the Dinkelbach method-based convex programming is proposed in the inner-layer to obtain the optimal power allocation, and Dinkelbach iterative method is applied again in the outer-layer for an optimal TS control strategy. A simplified but practical special case with equal time switching factor is also considered, where a low complexity joint resource allocation algorithm has been developed. Numerical results demonstrate the EE performance of the proposed strategy of jointly optimizing the power allocation and the TS factors. More importantly, compared to the conventional OMA approach, our findings have illustrated that significant EE gain can be achieved by our proposed algorithm, and this has confirmed the advantages of integrating SWIPT into NOMA systems. In addition, it has been shown in literature that the energy efficiency of NOMA cellular networks could be further improved with multi-carrier techniques, i.e., OFDMA. Therefore, it is worthy of studying the joint subcarrier scheduling and resource allocation for SWIPT-based multi-carrier NOMA cellular networks in the future.

\section{APPENDIX A}

\section{ProOF OF Proposition 2}

To demonstrate the concavity of the objective function shown in (19), we firstly suppose that $\theta_{k}=\sum_{i=k}^{K} P_{i}(\forall k \in$ $\mathcal{K}), \theta_{k+1}=\sum_{i=k+1}^{K} P_{i}(k=1,2, \ldots, K-1)$ and $\theta_{K+1}=0$. Thus, the objective function in (19) can be rewritten as

$$
\begin{aligned}
& \Lambda_{E E}(\boldsymbol{P}) \\
= & B \sum_{k=1}^{K} \alpha_{k} \log _{2}\left(\frac{\sigma^{2}+g_{k} \theta_{k}}{\sigma^{2}+g_{k} \theta_{k+1}}\right) \\
- & q\left(\zeta \theta_{1}+P_{C}-\eta \sum_{k=1}^{K}\left(1-\alpha_{k}\right) g_{k} \theta_{1}\right) \\
= & B \sum_{k=2}^{K}\left(\alpha_{k} \log _{2}\left(\sigma^{2}+g_{k} \theta_{k}\right)-\alpha_{k-1} \log _{2}\left(\sigma^{2}+g_{k-1} \theta_{k}\right)\right) \\
- & q\left(\zeta \theta_{1}+P_{C}-\eta \sum_{k=1}^{K}\left(1-\alpha_{k}\right) g_{k} \theta_{1}\right) \\
- & \alpha_{K} B \log _{2}\left(\sigma^{2}\right)+\alpha_{1} B \log _{2}\left(\sigma^{2}+g_{1} \theta_{1}\right) .
\end{aligned}
$$

Then the first-order derivative of $\Lambda_{E E}(\boldsymbol{P})$ can be denoted as follows

$$
\begin{aligned}
& \frac{\partial \Lambda_{E E}(\boldsymbol{P})}{\partial P_{m}} \\
= & \frac{B}{\ln 2} \cdot\left(\frac{\alpha_{1} g_{1}}{\sigma^{2}+g_{1} \theta_{1}}+\sum_{k=2}^{m}\left(\frac{\alpha_{k} g_{k}}{\sigma^{2}+g_{k} \theta_{k}}-\frac{\alpha_{k-1} g_{k-1}}{\sigma^{2}+g_{k-1} \theta_{k}}\right)\right) \\
- & q\left(\zeta-\eta \sum_{k=1}^{K}\left(1-\alpha_{k}\right) g_{k}\right) .
\end{aligned}
$$

Furthermore, we can acquire the second-order derivative of $\Lambda_{E E}(\boldsymbol{P})$ as follows

$$
\begin{aligned}
& \frac{\partial^{2} \Lambda_{E E}(\boldsymbol{P})}{\partial P_{m} \partial P_{l}} \\
= & -\frac{B}{\ln 2} \cdot \sum_{k=2}^{j}\left(\frac{\alpha_{k} g_{k}^{2}}{\left(\sigma^{2}+g_{k} \theta_{k}\right)^{2}}-\frac{\alpha_{k-1} g_{k-1}^{2}}{\left(\sigma^{2}+g_{k-1} \theta_{k}\right)^{2}}\right) \\
& -\frac{B}{\ln 2} \cdot \frac{\alpha_{1} g_{1}^{2}}{\left(\sigma^{2}+g_{1} \theta_{1}\right)^{2}},
\end{aligned}
$$

where $j=\min \{m, l\}$.

Let $H_{m}=\frac{\partial^{2} \Lambda_{E E}(\boldsymbol{P})}{\partial P_{m}^{2}}$. According to (56), it's obvious that $\frac{\partial^{2} \Lambda_{E E}(\boldsymbol{P})}{\partial P_{m} \partial P_{l}}=H_{m}$ if $l \geq m$ and $\frac{\partial^{2} \Lambda_{E E}(\boldsymbol{P})}{\partial P_{m} \partial P_{l}}=H_{l}$ if $l \leq m$. Therefore, the Hessian matrix $\mathbf{H}$ can be denoted as

$$
\mathbf{H}=\left(\begin{array}{cccc}
H_{1} & H_{1} & \ldots & H_{1} \\
H_{1} & H_{2} & \ldots & H_{2} \\
\vdots & \vdots & & \vdots \\
H_{1} & H_{2} & \ldots & H_{K}
\end{array}\right) .
$$

Then we define $\mathbf{Q}=-\mathbf{H}$. According to (57), the $k$-th order principal minor of $\mathbf{Q}$ is shown as follows

$$
\begin{aligned}
Q_{k} & =\left|\begin{array}{cccc}
-H_{1} & -H_{1} & \ldots & -H_{1} \\
-H_{1} & -H_{2} & \ldots & -H_{2} \\
\vdots & \vdots & & \vdots \\
-H_{1} & -H_{2} & \ldots & -H_{k}
\end{array}\right| \\
& =\left|\begin{array}{cccc}
-H_{1} & -H_{1} & \ldots & -H_{1} \\
0 & H_{1}-H_{2} & \ldots & H_{1}-H_{2} \\
\vdots & \vdots & & \vdots \\
0 & 0 & \ldots & H_{k-1}-H_{k}
\end{array}\right| \\
& =\left\{\begin{array}{l}
-H_{1}, k=1 \\
-H_{1} \prod_{i=2}^{k}\left(H_{i-1}-H_{i}\right), 2 \leq k \leq K
\end{array}\right.
\end{aligned}
$$

According to (56), we have $-H_{1}=\frac{B}{\ln 2} \cdot \frac{\alpha_{1} g_{1}^{2}}{\left(\sigma^{2}+g_{1} \theta_{1}\right)^{2}} \geq 0$. For $2 \leq i \leq K$, we have

$$
\begin{aligned}
H_{i-1}-H_{i} & =\frac{B}{\ln 2} \cdot\left(\frac{\alpha_{i} g_{i}^{2}}{\left(\sigma^{2}+g_{i} \theta_{i}\right)^{2}}-\frac{\alpha_{i-1} g_{i-1}^{2}}{\left(\sigma^{2}+g_{i-1} \theta_{i}\right)^{2}}\right) \\
& =\frac{B}{\ln 2} \cdot\left(\frac{\alpha_{i}}{\left(\frac{\sigma^{2}}{g_{i}}+\theta_{i}\right)^{2}}-\frac{\alpha_{i-1}}{\left(\frac{\sigma^{2}}{g_{i-1}}+\theta_{i}\right)^{2}}\right) .
\end{aligned}
$$


Since every terminal has the same harvested power constraint, we can infer that $\alpha_{i-1} \leq \alpha_{i}$ based on the constraint condition (21) and the assumption mentioned above, i.e., $g_{1} \leq g_{2} \leq \ldots \leq g_{K}$. Therefore, in (59) $H_{i-1}-H_{i} \geq 0$ holds and thus $Q_{k} \geq 0(\forall k \in \mathcal{K})$, which implies that $\mathbf{Q}=-\mathbf{H} \succeq \mathbf{0}$ and $\mathbf{H} \preceq \mathbf{0}$ [38]. As a consequence, we can conclude that the objective function (19) is concave in $\boldsymbol{P}$.

\section{APPENDIX B}

\section{PROOF OF PROPOSITION 4}

To demonstrate the EE in objective function (48) is monotonically increasing with respect to the time switching factor $\alpha$, we firstly let

$$
\begin{gathered}
\mathcal{X}=B \sum_{k=1}^{K} \log _{2}\left(1+\frac{g_{k} P_{k}}{\sigma^{2}+g_{k} \sum_{i=k+1}^{K} P_{i}}\right), \\
\mathcal{Y}=\zeta \sum_{k=1}^{K} P_{k}+P_{C}-\eta \sum_{k=1}^{K} g_{k} \sum_{i=1}^{K} P_{i} \\
\mathcal{Z}=\eta \sum_{k=1}^{K} g_{k} \sum_{i=1}^{K} P_{i} .
\end{gathered}
$$

And thus, (48) is simplifed as

$$
\lambda_{E E}(\alpha) \triangleq \frac{\mathcal{X} \alpha}{\mathcal{Y}+\mathcal{Z} \alpha}=\frac{\mathcal{X}}{\frac{\mathcal{y}}{\alpha}+\mathcal{Z}}
$$

Obviously, $\mathcal{X} \geq 0$ and $\mathcal{Z} \geq 0$ always hold; and in practice, we have $\mathcal{Y}>0$. Based on these, $\frac{\mathcal{Y}}{\alpha}$ as well as $\frac{\mathcal{Y}}{\alpha}+\mathcal{Z}$ decreases with respect to $\alpha$, and thus $\frac{\mathcal{X}}{\frac{\mathcal{Y}}{\alpha}+\mathcal{Z}}$, i.e., the objective function in (48), is monotonically increasing with respect to $\alpha$. This complete the proof of Proposition 4.

\section{REFERENCES}

[1] J. G. Andrews, S. Buzzi, W. Choi, S. V. Hanly, A. Lozano, A. C. K. Soong, and J. C. Zhang, "What will 5G be?" IEEE Journal on Sel. Areas in Commun., vol. 32, no. 6, pp. 1065-1082, June 2014.

[2] D. W. K. Ng, E. S. Lo, and R. Schober, "Wireless information and power transfer: Energy efficiency optimization in OFDMA systems," IEEE Trans. Wireless Commun., vol. 12, no. 12, pp. 6352-6370, Dec. 2013.

[3] Y. Liu, Z. Qin, M. Elkashlan, Z. Ding, A. Nallanathan, and L. Hanzo, "Non-orthogonal multiple access for 5G and beyond," Proceedings of the IEEE, vol. 105, no. 12, pp. 2347-2381, Dec 2017.

[4] Z. Ding, Z. Yang, P. Fan, and H. V. Poor, "On the performance of non-orthogonal multiple access in 5G systems with randomly deployed users," IEEE Sig. Process. Lett., vol. 21, no. 12, pp. 1501-1505, Dec. 2014.

[5] Z. Ding, X. Lei, G. K. Karagiannidis, R. Schober, J. Yuan, and V. K. Bhargava, "A survey on non-orthogonal multiple access for $5 \mathrm{G}$ networks: Research challenges and future trends," IEEE Journal on Sel. Areas in Commun., vol. 35, no. 10, pp. 2181-2195, Oct 2017.

[6] Z. Ding, Z. Yang, P. Fan, and H. V. Poor, "QoE-based resource allocation for multi-cell NOMA networks," IEEE Trans. Wireless Commun., vol. 17, no. 9, pp. 6160-6176, Sept. 2018.

[7] J. Zhu, J. Wang, Y. Huang, S. He, X. You, and L. Yang, "On optimal power allocation for downlink non-orthogonal multiple access systems," IEEE Journal on Selected Areas in Communications, vol. 35, no. 12, pp. 2744-2757, Dec. 2017.

[8] J. Wang, Q. Peng, Y. Huang, H. Wang, and X. You, "Convexity of weighted sum rate maximization in NOMA SYSTEMS, journal = IEEE Sig. Process. Lett., year $=$ Sept. 2017, volume $=24$, number $=9$, pages $=$ 1323-1327, annote $=, . "$
[9] R. Ruby, S. hong, H. Yang, and K. Wu, "Enhanced uplink resource allocation in non-orthogonal multiple access systems," IEEE Trans. Wireless Commun., vol. 17, no. 3, pp. 1432-1444, March 2018.

[10] Z. Chen, Z. Ding, X. Dai, and R. Zhang, "An optimization perspective of the superiority of 5G NOMA compared to conventional OMA," IEEE Trans. Signal Processing, vol. 65, no. 19, pp. 5191-5202, Oct. 2017.

[11] Y. Zhang, H. M. Wang, T. X. Zheng, and Q. Yang, "Energy-efficient transmission design in non-orthogonal multiple access," IEEE Trans. Veh. Techn., vol. 66, no. 3, pp. 2852-2857, March 2017.

[12] Z. Q. Al-Abbasi and D. K. C. So, "Resource allocation in non-orthogonal and hybrid multiple access system with proportional rate constraint," IEEE Trans. Wireless Commun., vol. 16, no. 10, pp. 6309-6320, Oct. 2017.

[13] H. Zhang, F. Fang, J. Cheng, K. Long, W. Wang, and V. C. M. Leung, "Energy-efficient resource allocation in NOMA heterogeneous networks," IEEE Wireless Commun., vol. 25, no. 2, pp. 48-53, April 2018.

[14] X. Lu, P. Wang, D. Niyato, D. I. Kim, and Z. Han, "Wireless networks with RF energy harvesting: A contemporary survey," IEEE Commun. Surv. Tut., vol. 17, no. 2, pp. 757-789, Secondquarter 2015.

[15] L. R. Varshney, "Transporting information and energy simultaneously," in Proc. IEEE Int. Symp. Info. Theory, July 2008, pp. 1612-1616.

[16] X. Zhou, R. Zhang, and C. K. Ho, "Wireless information and power transfer: Architecture design and rate-energy tradeoff," IEEE Trans. Commun., vol. 61, no. 11, pp. 4754-4767, Nov. 2013.

[17] R. Zhang and C. K. Ho, "MIMO broadcasting for simultaneous wireless information and power transfer," IEEE Trans. Wireless Commun., vol. 12, no. 5, pp. 1989-2001, May 2013.

[18] C. Song, J. Park, B. Clerckx, I. Lee, and K. J. Lee, "Generalized precoder designs based on weighted MMSE criterion for energy harvesting constrained MIMO and multi-user MIMO channels," IEEE Trans. on Wireless Commun., vol. 15, no. 12, pp. 7941-7954, Dec. 2016.

[19] S. Luo, J. Xu, T. J. Lim, and R. Zhang, "Capacity region of MISO broadcast channel for simultaneous wireless information and power transfer," IEEE Trans. Commun., vol. 63, no. 10, pp. 3856-3868, Oct. 2015.

[20] Y. Huang and B. Clerckx, "Relaying strategies for wireless-powered MIMO relay networks," IEEE Trans. Wireless Commun., vol. 15, no. 9, pp. 6033-6047, Sept. 2016

[21] Z. B. Zawawi, J. Park, and B. Clerckx, "Simultaneous wireless information and power transfer in a two-user OFDM interference channel," in Proc. Int. Symp. Wireless Commun. Sys. (ISWCS), Aug 2015, pp. 266-270.

[22] X. Zhou, R. Zhang, and C. K. Ho, "Wireless information and power transfer in multiuser OFDM systems," IEEE Trans. Wireless Commun., vol. 13, no. 4, pp. 2282-2294, April 2014.

[23] Q. Shi, L. Liu, W. Xu, and R. Zhang, "Joint transmit beamforming and receive power splitting for MISO SWIPT systems," IEEE Trans. Wireless Commun., vol. 13, no. 6, pp. 3269-3280, June 2014.

[24] W. Lu, Y. Gong, J. Wu, H. Peng, and J. Hua, "Simultaneous wireless information and power transfer based on joint subcarrier and power allocation in OFDM systems," IEEE Access, vol. 5, pp. 2763-2770, 2017.

[25] J. Xu, L. Liu, and R. Zhang, "Multiuser MISO beamforming for simultaneous wireless information and power transfer," IEEE Trans. on Signal Processing, vol. 62, no. 18, pp. 4798-4810, Sept 2014.

[26] H. Zhang, J. Du, J. Cheng, K. Long, and V. C. M. Leung, "Incomplete CSI based resource optimization in SWIPT enabled heterogeneous networks: A non-cooperative game theoretic approach," IEEE Trans. on Wireless Commun., vol. 17, no. 3, pp. 1882-1892, March 2018.

[27] H. Zhang, J. Du, J. Cheng, and V. C. M. Leung, "Resource allocation in SWIPT enabled heterogeneous cloud small cell networks with incomplete CSI," in 2016 IEEE Global Communications Conference (GLOBECOM), Dec 2016, pp. 1-5.

[28] J. Tang, D. K. C. So, A. Shojaeifard, and K. K. Wong, "Energy efficiency optimization with SWIPT in MIMO broadcast channels for internet of things," to appear in IEEE Internet of Things Journal, 2017.

[29] C. Psomas and I. Krikidis, "Successive interference cancellation in bipolar ad hoc networks with SWIPT," IEEE Wireless Communications Letters, vol. 5, no. 4, pp. 364-367, Aug 2016.

[30] P. D. Diamantoulakis, K. N. Pappi, Z. Ding, and G. K. Karagiannidis, "Wireless-powered communications with non-orthogonal multiple access," IEEE Trans. Wireless Commun., vol. 15, no. 12, pp. 8422-8436, Dec. 2016.

[31] P. D. Diamantoulakis, K. N. Pappi, G. K. Karagiannidis, H. Xing, and A. Nallanathan, "Joint downlink/uplink design for wireless powered networks with interference," IEEE Access, vol. 5, pp. 1534-1547, 2017. 
[32] G. He, L. Li, X. Li, W. Chen, L. L. Yang, and Z. Han, "Secrecy sum rate maximization in NOMA systems with wireless information and power transfer," in Proc. 9th Int. Conf. Wireless Commun. and Sig. Process. (WCSP), Oct 2017, pp. 1-6.

[33] Y. Liu, Z. Ding, M. Elkashlan, and H. V. Poor, "Cooperative nonorthogonal multiple access with simultaneous wireless information and power transfer," IEEE Journal on Selected Areas in Communications, vol. 34, no. 4, pp. 938-953, April 2016.

[34] T. N. Do, D. B. da Costa, T. Q. Duong, and B. An, "Improving the performance of cell-edge users in MISO-NOMA systems using TAS and SWIPT-based cooperative transmissions," IEEE Transactions on Green Communications and Networking, vol. 2, no. 1, pp. 49-62, March 2018.

[35] N. Jindal, S. Vishwanath, and A. Goldsmith, "On the duality of gaussian multiple-access and broadcast channels," IEEE Trans. Inf. Theory, vol. 50, no. 5, pp. 768-783, May 2004.

[36] D. W. K. Ng, E. S. Lo, and R. Schober, "Robust beamforming for secure communication in systems with wireless information and power transfer," IEEE Trans. Wireless Commun., vol. 13, no. 8, pp. 4599-4615, Aug. 2014.

[37] J. Tang, D. K. C. So, A. Shojaeifard, K. K. Wong, and J. Wen, "Joint antenna selection and spatial switching for energy efficient MIMO SWIPT system," IEEE Trans. Wireless Commun., vol. 16, no. 7, pp. $4754-4769$, July 2017.

[38] S. Boyd and L. Vandenberghe, Convex Optimization. Cambridge University Press, Cambridge, UK, 2004.

[39] W. Dinkelbach, "On nonlinear fractional programming," Management Science, vol. 13, pp. 492-498, Mar. 1967.

[40] L. Zhang, Y. Xin, and Y. C. Liang, "Weighted sum rate optimization for cognitive radio MIMO broadcast channels," IEEE Trans. Wireless Commun., vol. 8, no. 9, pp. 2950-2959, Jun. 2009.

[41] X. Huang and V. Y. Pan, "Fast rectangular matrix multiplication and applications," Journal of Complexity, vol. 14, no. 2, pp. 257 - 299, 1998. [Online]. Available: http://www.sciencedirect.com/science/article/ pii/S0885064X98904769

[42] J. J. M. Paul H. Calamai, "Projected gradient methods for linearly constrained problems," Mathematical Programming, vol. 39, no. 1, pp. 93-116, 1987.

[43] GreenTouch, Mobile Communications WG architecture doc2: Refference scenarios, May. 2013.

[44] I. Krikidis, S. Timotheou, S. Nikolaou, G. Zheng, D. W. K. Ng, and R. Schober, "Simultaneous wireless information and power transfer in modern communication systems," IEEE Communications Magazine, vol. 52, no. 11, pp. 104-110, Nov 2014.

[45] J. Tang, D. K. C. So, E. Alsusa, K. A. Hamdi, and A. Shojaeifard, "On the energy efficiency-spectral efficiency trade-off in MIMO-OFDMA broadcast channels," IEEE Trans. Veh. Tech., vol. 65, no. 7, pp. 51855199 , July 2016.

[46] J. Tang, D. So, E. Alsusa, K. A. Hamdi, and A. Shojaeifard, "Energy efficiency optimization with interference alignment in multi-cell MIMO interfering broadcast channels," IEEE Trans. Commun., vol. 63, no. 7, pp. 2486-2499, July 2015.

[47] 1 , "Resource allocation for energy efficiency optimization in heterogeneous networks," IEEE Journal on Sel. Areas in Commun., vol. 33, no. 10, pp. 2104-2117, Oct. 2015.

[48] J. Tang, D. K. C. So, E. Alsusa, and K. A. Hamdi, "Resource efficiency: A new paradigm on energy efficiency and spectral efficiency tradeoff," IEEE Trans. Wireless Commun., vol. 13, no. 8, pp. 46564669, Aug. 2014. 